Referencia para citar este artículo: González, V., Orcasita, L. T., Carrillo, J. P. \& Palma-García, D. M. (2017). Comunicación familiar y toma de decisiones en sexualidad entre ascendientes y adolescentes. Revista Latinoamericana de Ciencias Sociales, Niñez y Juventud, 15(1), pp. 419-430.

\title{
Comunicación familiar y toma de decisiones en sexualidad entre ascendientes y adolescentes*
}

\author{
VALENTINA GONZALEZ $Z^{* *}$ \\ Psicóloga Pontificia Universidad Javeriana de Cali, Colombia. \\ LINDA TERESA ORCASITA*** \\ Docente-investigadora pontificia Universidad Javeriana de Cali, Colombia. \\ JUAN PabLo CARRILLO \\ Psicólogo Pontificia Universidad Javeriana de Cali, Colombia. \\ Diana MarCela PaLMa-GarCía ${ }^{* * * * *}$ \\ Psicóloga Pontificia Universidad Javeriana de Cali, Colombia.
}

\section{Artículo recibido en febrero 5 de 2016; artículo aceptado en marzo 28 de 2016 (Eds.)}

- Resumen (descriptivo): Los individuos adolescentes aprenden de sexualidad a través de diferentes medios, pero la familia es uno de los primeros agentes socializadores y es la encargada, a través de la comunicación, de trasmitir información sobre ésta. Este estudio tiene como objetivo describir los procesos de comunicación que se establecen entre padres y madres con sus hijos e hijas adolescentes para entablar diálogos sobre sexualidad y su relación con la toma de decisiones, en dos familias pertenecientes a instituciones educativas de Cali. A través de esta investigación cualitativa encontramos que los procesos de comunicación, los temas abordados y los recursos empleados, están influidos por los conocimientos y percepciones de los ascendientes, en quienes la sexualidad aún es un tabú y tienen carencia de información y de estrategias de acompañamiento.

Palabras clave: Sexualidad, adolescentes, comunicación, toma de decisiones (Thesaurus Decs) ${ }^{1}$.

\section{Family communication and decision-making about sexuality between parents and adolescents}

- Abstract (descriptive): Adolescents learn about sexuality in different ways, yet parents are one of the first socializing agents who are in charge of transmitting information about sexuality through

\footnotetext{
* Este artículo de investigación científica y tecnológica presenta resultados del proyecto de investigación titulado: Procesos de comunicación entre padres e hijos adolescentes frente a la toma de decisiones en sexualidad en dos municipios priorizados por el Conpes 147. Código del proyecto: 00004250. Proyecto Financiado por la Pontificia Universidad Javeriana Seccional Cali-Colombia. La investigación inició en enero del 2016 y finalización en diciembre del 2016 fase III. Área del conocimiento: Psicología; subárea: Temas especiales.

** Psicóloga de la Pontificia Universidad Javeriana Cali. Correo electrónico: valen-gonzalez@hotmail.com

*** Magíster en Familia y psicóloga de la Pontificia Universidad Javeriana Cali. Docente e investigadora Miembro del Grupo de Medición y Evaluación Psicológica (GMEP) en la línea de Ciclo Vital Humano, Riesgo y Socialización Pontificia Universidad Javeriana Seccional CaliColombia. Correo electrónico: 1torcasita@javerianacali.edu.co

**** Psicólogo de la Pontificia Universidad Javeriana Cali. Correo electrónico: jpcarrillo22@gmail.com

***** Psicóloga de la Pontificia Universidad Javeriana Cali. Miembro del Grupo de Medición y Evaluación Psicológica (GMEP) en la línea de Ciclo Vital Humano, Riesgo y Socialización. Correo electrónico: dmpalma@javerianacali.edu.co

1 Estas palabras clave fueron tomadas del Thesaurus Decs (descriptores en Ciencias de la Salud) http://decs.bvs.br/cgi-bin/wxis1660.exe/ decsserver/
} 
communication. This in turn leads teenagers to develop their own set of representations of sexuality and decision-making. This study aims to describe the communication process established between parents and teenagers in relation to sexuality and, as a result, identify decision-making procedures in two families of students at secondary schools in the city of Cali, Colombia. Through this qualitative research it was found that communication processes, conversation topics and the resources used to address these topics were influenced by the perceptions and knowledge of the parents. The study identified that sexuality was still a taboo subject for these families and there were gaps in the information and accompaniment provided.

Key words: Sexuality, adolescents, communication, decision-making (Thesaurus Decs).

\section{Comunicação familiar e tomada de decisões em sexualidade entre pais/mães com crianças adolescentes}

- Resumo (descritivo): Os adolescentes aprendem sobre a sexualidade através de diferentes meios, mas a família é um dos primeiros agentes socializadores e são responsáveis, por meio da comunicação, transmitir informações sobre o tema. Este estudo teve como objetivo descrever os processos de comunicação estabelecidos entre pais, mães e filhos/as adolescentes para o diálogo sobre sexualidade e sua relação com a tomada de decisão em duas famílias pertencentes a instituições de ensino em Cali. Através desta pesquisa qualitativa constatou-se que os processos de comunicação, as questões abordadas e os recursos utilizados são influenciados pelo conhecimento e percepções dos pais, onde se considera que a sexualidade ainda é um tabu e que existem lacunas em termos de informação. Decs).

Palavras-chave: Sexualidade, adolescentes, comunicação, tomada de decisões (Thesaurus

\section{-1. Introducción. -2. Método. -3. Resultados. -4. Discusión. -Lista de referencias.}

\section{Introducción}

Los adolescentes en Colombia representan el $20.3 \%$ de la población. Según la Encuesta Nacional de Demografía y Salud (ENDS) (Profamilia, 2010), este grupo presenta una tendencia creciente en las tasas de fecundidad estimadas. Se reporta que el $14 \%$ de las mujeres entre 15 y 24 años de edad tuvo su primera relación antes de los 15 años, y el $61 \%$ antes de los 18 años. En relación con las infecciones de transmisión sexual (ITS), el 45\% de los nuevos casos de VIH corresponden a mujeres entre los 15 y los 24 años de edad, pero solo el 11\% de las mujeres tienen conocimientos sobre las ITS.

El Fondo de Poblaciones de las Naciones Unidas (UNFPA, 2012) señala que las razones del no uso de métodos preventivos, en este grupo poblacional, son la falta de conocimiento sobre los derechos básicos de reproducción y la falta de información sobre los anticonceptivos. La ausencia de información en ocasiones se relaciona con las barreras que aún existen para hablar sobre temas como la igualdad entre hombres y mujeres, los anticonceptivos y la prevención de las ITS. Esta entidad sostiene que los lazos que establecen los individuos jóvenes con su núcleo familiar, es uno de los factores más importantes para su salud y su desarrollo.

Por otra parte, Elliott (2010) menciona que la educación sexual debe ser parte de la educación integral del sujeto adolescente, pues promueve el desarrollo de una personalidad saludable y de una sexualidad vinculada a la salud, al amor y a la equidad. Esta educación debe transmitir herramientas de cuidado del cuerpo como un espacio de experiencia, expresión de sentimientos, emociones para entender, analizar y cuidar.

Es así como Bull, Della y Pariche (2011) mencionan la importancia que tienen los padres y las madres al momento de comunicarse con sus hijos e hijas en la etapa de adolescencia, pues en ese momento la persona joven se caracteriza por la búsqueda de su propia identidad, lo que genera que empiece a 
conocerse a sí misma e interactúe con nuevos pares. En esta investigación, comprendemos el proceso de comunicación como el intercambio de información y significados de los padres y las madres sobre la sexualidad, y cómo las interpretaciones que han elaborado a partir de su experiencia influyen en la transmisión de información a sus hijas y sus hijos. Esta comunicación cumple tres funciones básicas: la informativa o cognoscitiva, la reguladora y la afectiva (Garcés \& Palacio, 2010).

Uno de los temas influido por la familia es el de los intereses, las creencias, las actitudes y los valores que se vuelven una referencia y que intervienen en el abordaje de los aspectos relacionados con la sexualidad. Las figuras del padre y de la madre, así como el diálogo que se establece frente a las nuevas inquietudes que se suscitan por el adolescente, comienzan a tomar un rol importante en las decisiones y las elecciones que toman frente a sus experiencias y prácticas sexuales (Vargas, Henao \& González, 2007).

Algunas investigaciones previas relacionadas con la comunicación y la sexualidad entre padres y madres con sus hijos y sus hijas señalan que las conversaciones se centran en abordajes basados en el miedo a los embarazos no deseados y a las ITS, pero poco se habla sobre las experiencias sexuales (Kajula, Sheon, De Vries, Kaaya \& Aarø, 2014). Así mismo, se han reportado diferencias de género en las conversaciones que se establecen (Jones, 2010a, Sevilla \& Orcasita, 2014). Por ejemplo, en el estudio de Jones (2010b), se encontró que la comunicación en sexualidad ocurre generalmente con sus madres y contiene un discurso de valores y reglas que transmite una orientación moral para la actividad sexual.

Respecto a la comodidad para hablar de sexualidad del padre o la madre, se ha identificado que, desde la perspectiva de los adolescentes, en el caso de los padres se presentan mayores niveles de incomodidad que en el de las madres (Gaona, Montijo \& Díaz-Loving, 2013). Además, se encontró que las personas adolescentes no preguntan sobre las relaciones sexuales por vergüenza $(57 \%)$ y porque buscan a otras personas para hablar de ese tema (26\%) (Oglea, Glasiera \& Rileya,
2008). Por su parte, en un estudio estadounidense se evidenció que el género influye en el grado de comunicación, y que son las madres quienes más frecuentemente informan sobre las consecuencias negativas de la vida sexual activa y sobre los métodos de planificación. Por su parte, los padres no conservadores reportan que presentan un reconocimiento positivo sobre la efectividad, seguridad y uso de métodos anticonceptivos, a diferencia de los padres conservadores, que tienen una creencia negativa sobre el uso de anticonceptivos y establecen una mayor comunicación orientada hacia las consecuencias negativas de la vida sexual (Swain, Ackerman \& Ackerman, 2006).

Respecto a la toma decisiones que realizan los adolescentes al iniciar su vida sexual, autores como Langer, Zimmerman, Warheit y Ducan (citados en Vargas et al., 2007) señalan que en esta etapa se enfrentan a la responsabilidad de tomar decisiones que tienen consecuencias importantes para su salud. Desde la perspectiva del desarrollo, las personas adolescentes que están en una edad más temprana, tienden a tomar decisiones teniendo en cuenta la opinión de sus padres y madres. Sin embargo, a medida que avanza la adolescencia, las decisiones están mediadas por la opinión del grupo de referencia, y hasta el final de la adolescencia las decisiones empiezan a tomarse con base en un criterio personal (Lavielle-Sotomayor, Jiménez-Valdez, Vázquez-Rodríguez, AguirreGarcía, Castillo-Trejo \& Vega-Mendoza, 2014, Vargas et al., 2007).

A partir de lo mencionado, en esta investigación tuvimos como objetivo central analizar los procesos de comunicación que se establecen entre padres y madres con sus hijos y sus hijas adolescentes para entablar diálogos sobre sexualidad, y su relación con la toma de decisiones, en dos familias de conformación nuclear pertenecientes a instituciones educativas de la ciudad de Cali, Colombia.

\section{Método}

\section{$\underline{\text { Diseño }}$}

El tipo de estudio que empleamos en la investigación fue de corte cualitativo. Nuestro propósito fue reconstruir la realidad 
interpretando y dando significado a las acciones de los sujetos en un ambiente natural (Bonilla \& Rodríguez, 1997).

\section{Participantes}

La población estuvo conformada por dos familias de tipología nuclear que tenían hijos e hijas adolescentes. En total, entrevistamos a dos padres, a dos madres y a dos adolescentes. Los padres y las madres tenían entre 38 y 45 años de edad. Los adolescentes tenían 16 años de edad y eran un muchacho y una muchacha.

\section{Instrumento}

Para el desarrollo de la investigación, utilizamos una entrevista semiestructurada con acudientes (madre padre) y sus hijos e hijas, que tuvo como propósito comprender, conocer $\mathrm{y}$ entender lo que piensa o siente una persona en un tema o situación particular. A través de ella analizamos lo que la persona dice y hace a través de la guía de preguntas construida con las categorías de análisis iniciales. Empleamos un tiempo de una hora aproximadamente con cada participante; en cuanto al lugar, realizamos las entrevistas en instituciones educativas.

\section{Procedimiento}

Contactamos dos colegios de Cali de carácter público y privado que participaban en el marco del proyecto macro en el cual realizamos la presente investigación. Posteriormente citamos a las familias interesadas en participar en el estudio para que conocieran los objetivos de la investigación, y para que dieran el consentimiento informado orientado a participar en ella. Los participantes fueron dos familias conformadas por tres integrantes cada una, donde priorizamos un análisis en profundidad que permitiera adentrarse a la realidad del caso de ambas familias. Después de la obtención de la información a través de las entrevistas, realizamos la transcripción de éstas e hicimos un proceso de categorización de los datos por medio del programa Atlas.Ti 6.2. Finalmente, al tener la categorización de cada entrevista, escribimos los resultados y la discusión, teniendo como estrategia de análisis el método Consensual Qualitative Research (CQR). Este método es un consenso que se realiza entre los investigadores e investigadoras al realizar la lectura de las entrevistas y la categorización de los datos, con el objetivo de tener una mirada legible a las narrativas comunes de los participantes desde su realidad, y así evitar posibles sesgos (Hill, Knox, Thompson, Nutt, Hess \& Ladany, 2005, Santiago, Gómez, Etchebarne \& Roussos, 2011).

\section{Consideraciones Éticas}

Para el presente estudio tuvimos en cuenta los Artículos 15 y 16 del Capítulo 1 de la Resolución No. 008430 de 1993, que establece las normas científicas, técnicas y administrativas para la investigación en salud. Los artículos mencionados establecen la información y los requisitos que se deben tener en cuenta para elaborar el consentimiento informado.

\section{Resultados}

A continuación, presentamos los resultados de la categoría procesos de comunicación (temas-recursos, conocimientos, percepciones, significado frente al diálogo) que establecen los padres y las madres al establecer un diálogo con su hijo o su hija.

\section{Temas y recursos "Como empezar a hablar de sexualidad" \\ La subcategoría temas y recursos se} refiere a las estrategias y los espacios que los padres y las madres emplean para poder acercarse a su hijo o hija adolescente y tener un diálogo sobre algún tema de sexualidad, según las interpretaciones que aquellos han elaborado. Se presenta una diferencia entre las dos familias en los siguientes aspectos: para la familia 2, la sexualidad es un tema normal y necesario, mientras que la familia 1 considera que la sexualidad también incluye, aparte de lo fisiológico, los sentimientos y los valores. La familia 2 percibe que, aunque la sexualidad es normal, aún es un tabú, lo que hace que se les dificulte entablar conversaciones con su hijo. Para la familia 1, se debe dialogar sobre sexualidad únicamente cuando su hijo y su hija estén en la edad adecuada. La estrategia más frecuente es buscar al adolescente cuando se encuentre en una relación y poder hablar desde 
la experiencia del padre y la madre. Otro recurso identificado es buscar ayuda de otra persona o institución para que los asesore sobre cómo acercarse y empezar a hablar de sexualidad:

(...) la verdad muy poco, hablamos mucho con él pero sobre otros temas, pero abordar sobre sexo realmente casi no hay comunicación, siempre la sexualidad no ha dejado de ser como un tabú y más cuando el padre o madre debe hablar de estos temas, aún existe mucho tabú y uno no sabe cómo abordarlo (familia 2).

Mi estrategia es lo que mi mujer habla con ella yo me quedo tranquilo porque soy hombre y claramente mi hija no me va a contar sus cosas, además a uno como padre no está preparado que su hija le cuente ciertas cosas porque para uno sigue siendo la niña (Familia 1).

Entre otros temas prevalece hablar de protección, de métodos de planificar y de embarazos no deseados. Encontramos que los padres y las madres de ambas familias abordan el tema de sexualidad con sus hijos e hijas pero lo consideran un tabú. Aunque ambas madres hablan con sus hijos e hijas, existen diferencias en los discursos que tienen. Para la madre 2, el tema más importante es el embarazo y la protección; mientras que para la madre de la familia 1, es importante que su hija esté preparada para su primera vez. En cuanto a los recursos hay una diferencia entre las madres: en el caso de la madre 2, hasta el momento ha empleado llevar a su hijo a unas clases de educación sexual, debido a que se le dificulta el diálogo con él; contrario a la madre 1 quien conversó con su hija en el momento en que ella tuvo su primer novio.

Conocimientos: "Y qué sabemos sobre sexualidad"

En la subcategoría conocimientos exploramos las conceptualizaciones y representaciones de los padres y las madres sobre sexualidad que finalmente trasmitirán a sus hijos e hijas. Los conocimientos que tienen los padres y las madres de ambas familias sobre sexualidad están muy influenciados por la noción de embarazo, las enfermedades de trasmisión sexual y los preservativos. Para la familia 2, la sexualidad es un aspecto biológico, pero sienten que ellos tienen poco conocimiento y por eso no se atreven a dialogar con sus hijos. Por otra parte, consideran que la época ha cambiado y que ahora las personas jóvenes saben más que ellos, por lo cual no se sienten preparados para abordar el tema de la sexualidad. Por el contrario, la familia 1 manifiesta que la sexualidad no es solo una parte íntima sino que implica cambios en el cuerpo, manifestaciones de cariño; involucra más aspectos y cada persona le otorga un significado. Además, los conocimientos que tienen se relacionan con los temas y la información que le van a trasmitir a sus hijos e hijas según la etapa donde se encuentren (niñez, adolescencia).

Lo que pasa es que sexualidad no es solo la parte íntima, la sexualidad es la comunicación, es el rosarse, el tocarse, es tener una relación con la persona que sea no necesariamente debe ser íntimo... sexualidad abarca el cuerpo, los sentimientos, valores, etc. (familia 1).

\section{Percepciones "Los tiempos cambian"}

Exploramos las creencias o ideas que han elaborado los padres y las madres sobre sexualidad y cómo influyen en el diálogo con sus hijos y sus hijas. En las dos familias (padremadre), hay la creencia de considerar que la sexualidad es un tabú. Los padres y las madres han elaborado la idea de que la ausencia de respeto, la responsabilidad y el cuidado, son factores que pueden terminar en un embarazo no deseado o en enfermedades. La familia 2 percibe que hay una brecha generacional con sus hijos que no les permite la comunicación, dado que la representación de sexualidad es diferente en ambas generaciones. En la familia 1 , se reconoce que la sexualidad ha tenido cambios y que actualmente los jóvenes cuentan con fuentes de información para resolver dudas, como la Internet, pero consideran que es conveniente una comunicación guiada por los padres y las madres para que sean la primera fuente de información que busquen sus hijos y sus hijas.

(...) para mí es importante que él sepa sobre las enfermedades, ahora se ve mucha cosa y los muchachos no piensan 
en el riesgo, solo en el momento. El problema es que ellos piensan que uno les habla para molestarlos y uno la verdad también está desinformado de muchas cosas (Familia 2).

\section{Dificultades y fortalezas "Cómo empezar vs. experiencia"}

En la subcategoría dificultades y fortalezas, indagamos sobre cómo la sexualidad producía dificultades o fortalezas, de acuerdo con la presencia o la ausencia de los diálogos que pueden tener los padres y las madres con sus hijos y sus hijas, para identificar la forma como se percibe el padre o la madre, cuándo reconoce que tiene habilidades para abordar el la sexualidad, o qué produce la dificultad de hablar del tema. En ambas familias, las mayores dificultades son el temor a no saber abordar un tema y que los hijos y las hijas acudan a otras fuentes de información. Las dificultades que tienen los padres para hablar sobre sexualidad son: (a) no hay comunicación; (b) no saben cómo abordar a su hijo o su hija; (c) no hay suficientes conocimientos; (d) no hay confianza; (e) los cambios en la adolescencia dificultan hablarles del tema; (f) distanciamiento con el hijo o la hija que dificulta el diálogo; (g) incertidumbre de que no sean escuchados por sus hijos y sus hijas, y (h) diferencias de género en las conversaciones.

En las fortalezas, las dos familias, cuando los padres y las madres encuentran que tienen dificultades para abordar el tema, emplean estrategias para darles información sobre sexualidad a sus hijos y sus hijas; consideran que hablar desde la experiencia es un puente para acercarse al adolescente. Reconocen que sí pueden tener un diálogo sobre otros aspectos $y$, por tanto, el que exista un vínculo entre ellos es una fortaleza que deben trabajar para abordar la sexualidad.

\section{Discurso según el género}

"A mí me queda más fácil hablar con mi hijo porque soy..."

Esta categoría hace referencia a la diferencia según el discurso expuesto por el padre y la madre en lo relacionado con la sexualidad, para identificar cómo el rol puede generar dinámicas de diálogo con el hijo o la hija, y las construcciones de los padres y las madres. En las dos familias, las madres tienen más contacto con sus hijos o hijas para dialogar sobre sexualidad; mientras que los padres hablan de otros temas o no hablan con ellos. En la familia 2, la madre tiene más acercamiento con su hijo, pero sobre sexualidad no hay una suficiente confianza, según dice ella. El padre no habla sobre el tema y conversa con su hijo de temas del colegio y de la vida. Mientras tanto, en la familia 1 la madre está empezando a dialogar sobre la sexualidad con su hija, y el padre no tiene un acercamiento con ella pues considera que por ser hombre debe distanciarse $\mathrm{y}$ hay temas que no debe abordar.

(...) yo no me siento preparado de hablar de ese tema porque ella es mujer, no hay un vínculo de confianza, porque soy hombre y claramente mi hija no me va a contar sus cosas además uno como padre no está preparado para que su hija le cuente ciertas cosas porque para uno sigue siendo la niña (familia 1).

(...) uno como hombre lo puede orientar un poco más a diferencia de la mamá, porque los dos somos hombres y yo le puedo hablar de mis experiencias (familia 2).

(...) como a mí me genera algo de temor los cambios que tiene y él es muy cerrado para hablar con uno, a veces se dificulta una conversación porque uno no sabe cómo abordarlo (familia 2).

Como se observa, en la familia 2 hay una dificultad de no saber cómo acercarse a su hijo, aunque el padre reconoce que por ser hombre podría hablar desde la experiencia con el joven; mientras que el padre de la familia 1, no habla con su hija porque es mujer y por ese motivo tiene más confianza con su mamá. Las madres de ambas familias hablan con su respectivo hijo o hija, aunque reconocen que en su adolescencia nunca les hablaron de sexualidad. Por eso, encontramos que la madre de la familia 2 considera que la sexualidad es un tabú, que no tiene suficiente información y que se le dificulta mantener una conversación con su hijo; mientras que la madre de la familia 1, aunque a ella no le 
hablaron de sexualidad, reconoce que no quiere repetir la misma historia, por eso cuando habla con su hija enfatiza que la sexualidad no es solo sexo y además conversa con su esposo sobre la educación de su hija.

\section{Contexto "la adolescencia ha cambiado"}

En la subcategoría contexto, se buscaba indagar sobre cómo la cultura influye en lo que los padres transmiten al hablar con su hijo o su hija. Ambas familias coinciden en que las generaciones han cambiado y que ellos vivieron una sexualidad muy distinta a la de sus hijos. Por lo tanto, al ser padres, quieren hacer cambios y no repetir lo que ellos vivieron. Así mismo, coinciden en que a su generación nunca se le habló de sexualidad. Finalmente, los padres reconocen que el hogar debe ser la primera fuente de información que sus hijos deben buscar para aclarar dudas sobre la sexualidad.

"para poder trasmitir lo que uno sabe y ellos tenga algo de conocimiento, si yo sé que la sexualidad es algo necesario en el hombre, pero por los cambios que ahora uno ve en la sociedad él debe cuidarse, de pronto lo que le diga a él le puede servir y me tendría algo de confianza para buscarme a mí y no a otra persona" (Familia 2).

\section{Toma de decisiones "Ya soy adolescente y soy grande" \\ Los resultados de la toma de decisiones} (expectativa-vivencia, sentimientos asociados al estilo de conversación, fortalezas- debilidades, discurso según el rol y contexto) manifestado por los adolescentes, fue el contraste para identificar la influencia que tiene la comunicación en la elección que toma el joven respecto a la sexualidad. La subcategoría expectativa $y$ vivencia, hace referencia a cómo el adolescente a partir de sus construcciones, empieza a hacer elecciones alrededor de los temas que abarca la sexualidad (pareja, protección, relaciones sexuales). En esta categoría se analizaron los relatos de los dos adolescentes, hombre y mujer de 16 años. Como resultado encontramos que en el adolescente (hombre, familia 2), considera la sexualidad en relación con el cuerpo como algo biológico donde el hombre debe protegerse y la mujer planificar. En contraste, la adolescente (mujer, familia 1) considera que la sexualidad, aparte de ser algo biológico, implica cuidar su cuerpo, responsabilidad y respeto. Finalmente, en los dos adolescentes a partir de sus construcciones guiados por sus padres, instituciones o pares, elaboran sus propios intereses, creencias y actitudes para tomar decisiones sobre su sexualidad. Esto se puede notar en los siguientes discursos.

"Sexualidad es cuidar de mi cuerpo, ser responsable al momento de iniciar una relación sexual, involucra a mi novio y el respeto que tengo por mí y la otra persona". (Familia 1)

"Como hombre protegerse y en el caso de la mujer que planifique." (Familia 2)

"Porque te dan una base, un conocimiento que uno no tiene y ellos por experiencia ya vivieron en este caso mi mamá, a uno se le va grabando lo que te dicen además lo que hablo con mi mamá al final me sirve a ser responsable, mas autónoma no dejarme llevar por el momento, darme mi lugar como mujer ellos me han dado base". (Familia 1)

Es que sexualidad implica hablar del cuerpo y esas cosas ya son muy personales, además si uno ya tiene relaciones uno se siente que es grande porque aparte estas siendo responsable con tus cosas. (Familia 2)

\section{Sentimientos asociados al estilo de conversación "Me gustaría saber qué opina" \\ La subcategoría "sentimientos asociados} al estilo de conversación”, hace referencia a la presencia o ausencia de diálogos, que se presentan entre los adolescentes y sus padres, cómo pueden influir en sus ideas frente a la sexualidad y las emociones, sentimientos que empiezan a construir. Se obtuvo en los resultados, que el adolescente hombre presenta un diálogo con su madre en el que tocan algunos temas de sexualidad y con su papá los diálogos se le limitan a los deberes y obligaciones. Por otra parte, piensa que para sus padres la sexualidad aún puede ser un tabú y que les da pena tocar este tema, aunque habla con su madre no puede compartir sus experiencias porque es mujer, por 
esa razón le gustaría que su papá se acercara pero afirma que no hay confianza. En el caso de la adolescente mujer, tiene conversaciones con su madre pero con su papá no dialoga debido a quele genera pena porque es hombre. La joven expresa lo que la razón por la que puede hablar con su mamá es que siente que ella es un apoyo y tiene conocimientos conocer acerca de la sexualidad, aun así reconoce que le gustaría escuchar la opinión de su papá en algún momento, pero no sabe cómo iniciar un diálogo con él. A continuación, se mostrarán algunos discursos expuestos por los adolescentes.

"yo no hablo con mi papá, ¡qué pena! él es hombre que le voy a decir" (Familia 1). "A veces me da hasta pereza porque mi papá no me habla de más temas, él es como la regla de la casa, hay muchas cosas que no le cuento porque después empieza hablarme de la moral y qué pereza" (familia 2).

"Ella no me decía nada y yo tenía muchas dudas, por ejemplo, nunca había tenido un novio, quería que mi mamá me hubiera hablado antes de qué es una relación, ella ya lo hizo, sino que creo que se demoró" (Familia 1).

"Mi mamá o las caras que me hace cuando me pregunta, es que a lo bien no tengo confianza con ellos yo siento que a mis papás también les parece incomodo hablar de sexualidad" (Familia 2).

\section{Discusión}

El objetivo fue describir los procesos de comunicación que se establecen entre padres y madres con sus hijos y sus hijas adolescentes para abordar temas de sexualidad. Encontramos que en las dos familias se reconoce la importancia de hablar sobre sexualidad, pues los padres consideran pertinente que sus hijos tengan conocimientos sobre este tema. Al iniciar un diálogo, este está mediado por ciertos discursos, percepciones e interpretaciones que los padres han elaborado y que influyen en la transmisión de información sobre sexualidad. Al respecto Jones (2010a) y Uribe y Rodríguez (2014) señalan que en estos diálogos entre padres e hijos habrá una trasmisión de valores, normas y opiniones que generan en los adolescentes una construcción frente a la sexualidad.

En relación con los procesos de comunicación, los padres buscan diferentes estrategias, como esperar que su hijo inicie una relación de pareja, hablar desde la experiencia o buscar ayuda de instituciones. Como también se señaló en el estudio de Shiferaw, Getahun y Asres (2014), cuando se acercan a sus hijos o han pedido ayuda externa para que hablen con el adolescente, se abordan temas en los que aún prevalece el del embarazo, las ITS y los métodos de planificación. Por tanto, estos resultados se suman a investigaciones previas, como las de Swain et al. (2006), Manu, Mba, Asare, OdoiAgyarko y Asante (2015) y Sevilla, Sanabria, Orcasita y Palma-García (2016), que muestran que aunque exista una comunicación entre padres e hijos, aún se brinda información sobre las consecuencias negativas de la vida sexual, por lo cual sigue educando sobre sexualidad mediante un abordaje biológico.

Los conocimientos que presentan los padres frente a la sexualidad están mediados por las representaciones que han elaborado y así son trasmitidos a sus hijos. Los padres reconocen que aún les falta información, pues a ellos no les suministraron la educación que ahora puede existir frente a la sexualidad. Como lo mencionan Lozano, Consuelo y Solís (2009), es fundamental que exista una educación que posibilite poder hablarlo, para poder difundir conocimiento y que el joven adquiera percepciones y construya significados frente a este tema. Esto implica que, según Molina, Torrivilla y Sánchez (2011), la educación sexual también debe incluir a los padres, para que puedan trasmitir conocimientos con naturalidad al momento de comunicarse con sus hijos. Para Elliott (2010), comunicarse facilita comprender el significado de la sexualidad y que se fortalezcan las habilidades para tener conversaciones abiertas y cómodas frente al tema.

A partir de la exploración de las percepciones que tienen los padres frente a la sexualidad, encontramos que están relacionadas a las creencias o ideas que elaboran frente a ella; en el caso de la investigación, hay una percepción de considerar la sexualidad como 
un tabú, donde se hace una relación de la sexualidad con el embarazo o enfermedades, lo que genera que los diálogos que se establecen con sus hijos promuevan el respeto, el cuidado y la responsabilidad. Las percepciones que han elaborado los padres son trasmitidas en un proceso de comunicación $\mathrm{y}$, como lo señala López, Murgui, Musitu y Moreno (2008), cuando se establece un diálogo con el otro, hay una trasmisión de lo que se piensa, se siente, y se comparten creencias, significados.

En relación con la toma de decisiones relacionadas con la sexualidad, se encuentra que influyen, en el caso de las dos familias, la falta de conocimiento, los cambios del adolescente, el sexo, y el no saber cómo iniciar la conversación con su hijo o su hija. Finalmente, esto crea condiciones para que se dé o no se dé na comunicación entre el padre, la madre y el hijo o la hija. De acuerdo con lo planteado por Campo-Arias (2009), en las dinámicas de los espacios de socialización, cuando el joven o la joven crece y empieza a explorar la sexualidad, ocurre un distanciamiento con los padres.

En conclusión, tal como ha sido señalado por Sevilla et al. (2016) y Kajula et al. (2014), las principales barreras para comunicarse son la percepción de los padres de no tener suficiente información y de no saber cómo abordar el tema. Estas conversaciones están mediadas por la representación de que la sexualidad es un tema tabú y por estereotipos de género.

Respecto a la fortaleza mencionada por los padres, la experiencia es uno de los medios para acercarse a los hijos y trasmitirles lo que ellos conocen sobre la sexualidad. Otra cualidad que se identificó es el papel de las madres, pues son ellas quienes abordan a sus hijos o sus hijas para establecer diálogos sobre la sexualidad. Es preciso tener en cuenta que las experiencias sobre sexualidad que los padres vivieron tienen relación con la época, la crianza y el aprendizaje, lo que para los y las adolescentes puede ser una barrera o un recurso para que se inicie el diálogo.

Por otro lado, lo que piensa cada padre o madre sobre la sexualidad, lo que logra trasmitir, los mecanismos que busca para hacerlo está mediado por su rol (Seloilwe, Magowe, Dithole $\&$ Lawrence, 2015). Este es un punto en el que hay una diferencia entre el discurso de las madres y los padres. Lo encontrado en la investigación, en el caso de ambas familias, es que el padre no logra tener una conversación sobre sexualidad y aborda otros temas con su hijo. La madre es quien presenta cercanía con su hijo o su hija. Por ejemplo, en la familia 1, la madre tiene un acercamiento con su hija porque es mujer y esto hace que pueda haber confianza entre ellas. En la familia 2, la madre que tiene un hijo varón, compartir más tiempo con él permite abordarlo para abarcar algunos temas de sexualidad. Como lo afirma Jones (2010c), en los discursos presentados entre padres e hijos, se establece una relación de mujer-madre, hombre-padre; cada relación se caracteriza por discursos según el sexo. En esta investigación, se presenta lo opuesto, en la familia 2, que tienen un hijo, el padre no abarca con él ningún tema de sexualidad, hasta el momento no se atreve hablar del tema. Esta dificultad del padre para entablar conversaciones sobre sexo ya ha sido reportado en la literatura por Sevilla et al. (2016) y Kajula et al. (2014).

En la investigación algunos padres reconocían que el contexto donde la persona vive su niñez, su adolescencia o su juventud, determina el significado que se le otorgue a la sexualidad y, dependiendo de ese significado, así mismo transmitirá su conocimiento a otra persona. Como se mencionó, poder trasmitir algo sólo ocurre cuando se comunica en un lenguaje verbal o no verbal, es por eso que en el momento en que los padres dialogan con sus hijos de acuerdo con las interpretaciones que han elaborado según el contexto (político, religioso, económico), aparece un intercambio de información, significados y sentido para comprender el mundo. Los padres transmiten las percepciones que tienen de sexualidad de acuerdo con lo vivido, y los hijos lo interpretarán, para darle un significado propio de su sexualidad (Bavelas, Jackson \& Watzlawick, 1990). En esa construcción el adolescente o la adolescente empieza a desarrollar una identidad, a tomar nuevas ideas, buscar compañía de su mismo sexo, tener interés y curiosidad por otra persona $\mathrm{y}$, en el ámbito psicológico, aparecen cambios en la capacidad cognitiva, en la habilidad para relacionarse con el medio, la familia, los 
amigos, el colegio y la sociedad. Entonces, los y las adolescentes toman nuevos ideales y consolidan nuevas identidades (Gómez, 2008).

Como se mencionó arriba, aunque los padres tienen dificultades para establecer diálogos sobre sexualidad, buscan estrategias para entablar una conversación ya sea con palabras, gestos y miradas para que sus hijos perciban lo que ellos intentan trasmitir. Esto se conecta con lo propuesto por Gallego (citado por Garcés y Palacios, 2010) que propone la presencia de un proceso simbólico dentro del sistema familiar, donde habrá una serie de mensajes verbales y no verbales que incluye percepciones, sentimientos y saberes de cada miembro de la familia.

Conviene señalar que el contexto sociocultural tiene una influencia en la toma de decisiones por el adolescente. Aquí, se encontró que los dos adolescentes empiezan a tomar decisiones de acuerdo con lo que piensan que es la sexualidad. Es por eso que no observamos que los jóvenes tomaran decisiones para cumplir las expectativas de su padre y su madre, lo cual puede significar que empiezan a tener conocimientos o información suministrada por su familia o por otra red de apoyo, lo que favorece el conocimiento de los jóvenes mediada por conocimientos, creencias, metas, expectativas, valoraciones, intereses, actitudes y normas sobre sí mismo y sobre la sexualidad, que serán una guía al tomar una decisión (Vargas et al., 2007).

Para concluir, se reconoce la importancia de hablar sobre sexualidad en las familias, pues es un sistema de apoyo en el que se transmiten conocimientos, valores, actitudes respecto al mundo, es decir, que las interpretaciones hechas por los padres tendrán una influencia para el adolescente en la elaboración de sus propias creencias, conocimientos, actitudes y valores. Por esta razón, es preciso que estos diálogos reconozcan las funciones de la sexualidad en la vida de las personas adolescentes: la biológica que expresa los aspectos fisiológicos, el aspecto erótico, relacionado con la búsqueda de placer, y la comunicativa, entendida como los procesos de interacción con los otros que incluye el lenguaje, la expresión, la negociación y los comportamientos.
En este estudio presentamos algunas limitaciones como contar con una muestra pequeña que impide la generalización de los resultados, así como la disposición de otras familias en participar, teniendo presente que es un tema de gran susceptibilidad para algunos padres y madres o en algunos casos para los sujetos adolescentes al momento de ser entrevistados por temor a los comentarios que se generen al abordar el tema.

Finalmente, es importante desarrollar desde otras perspectivas líneas futuras de investigación acción, enfocadas a los padres, madres y cuidadores de las personas adolescentes, donde se trabaje en torno a la educación sexual, estrategias para generar diálogos abiertos y flexibles que permitan el intercambio de conocimientos. Como lo menciona la UNFPA (2012), es necesario que los adolescentes y las adolescentes tengan un conocimiento sobre su sexualidad, brindándoles recursos en la intervención con programas orientados a partir de sus necesidades, lo cual favorece condiciones de bienestar en la calidad de vida. Esto implica que se debe trabajar mediante esfuerzos mancomunados entre varios actores que involucran activamente a los padres y las madres, pues la ausencia de formación y potenciación de habilidades está haciendo que estos no tengan recursos para iniciar una conversación con sus hijos o sus hijas, o no cuenten con los suficientes conocimientos para ser una guía frente al tema de la sexualidad. Es preciso fortalecer el trabajo interdisciplinar entre los psicólogos y psicólogas, los educadores y educadoras, y los médicos y médicas, en función de promover una salud sexual y crear líneas de acción que influyan en la información que se les suministra a los adolescentes y a los padres y las madres, y fortalecer los diálogos que hay entre ascendientes y descendientes. Si las instituciones solo se basan en informar al joven, los padres y las madres percibirán que sus hijos y sus hijas conocen más del tema, por lo que se requiere crear estrategias con los ascendientes, para desarrollar habilidades y fortalezas en relación con los procesos de comunicación y sobre la sexualidad. Adicionalmente, es preciso incluir en la investigación temas relacionados con: género, diversidad sexual, ciclo vital y 
enfoque de derechos en SSR. Es importante articular estas acciones a las entidades territoriales que trabajan en programas de SSR en los entornos de salud y educación, como las escuelas de padres y madres ya existentes en las instituciones.

\section{Lista de referencias}

Bavelas, J., Jackson, D. \& Watzlawick, P. (1990). Algunos axiomas exploratorios de la comunicación. Teoría de la comunicación humana. Barcelona: Herder.

Bonilla, E. \& Rodríguez, P. (1997). Recolección de datos cualitativos. Más allá del dilema de los métodos, La investigación en ciencias sociales. Bogotá, D. C.: Norma.

Bull, M., Della, I. \& Pariche, A. (2011). Factores comunicativos desencadenantes del aborto en la adolescencia. Contribuciones a las ciencias sociales. Recuperado de: http:// www.eumed.net/rev/cccss/20/smz.pdf

Campo-Arias, A. (2009). Relaciones sexuales en adolescentes colombianos y la implicación para la salud pública: Una revisión de prevención y algunas variables asociadas. MedUnab, 12 (2), pp. 86-89.

Elliott, S. (2010). Parents Constructions of Teen Sexuality: Sex Panics, Contradictory Discourses, and Social Inequality. Symbolicinteraction, 33 (2), pp. 191-212.

Doi: 10.1525/si.2010.33.2.191.

Fondo de Población de las Naciones Unidas-UNFPA (2012). Servicios de Salud Amigables para Adolescentes y Jóvenes. Recuperado de: http://unfpa. org.co/wp-content/uploads/2013/09/ modelosamigables2.pdf

Gaona, S., Montijo, S. \& Díaz-Loving, R. (2013). El papel de los padres en la salud sexual de sus hijos. Acta de Investigación Psicológica, 3 (1), pp. 956-968.

Garcés, M. \& Palacios, J. (2010). La comunicación familiar en asentamientos subnormales de Montería (Colombia). Psicología desde el Caribe, 25, pp. 1-29.

Gómez, C. (2008). Adolescencia y Familia: Revisión de la Relación y Comunicación como Factores de Riesgo y Protección.
Revista Intercontinental de Psicología y Educación 10, pp. 105-122.

Hill, C., Knox, S., Thompson, B., Nutt, E., Hess, S. \& Ladany, N. (2005). Consensual Qualitative Research: An Update. Journal of Counseling Psychology, 52 (2), pp. 196205. Doi: 10.1037/0022-0167.52.2196.

Jones, D. (2010a). Sexualidad adolescente, la primera relación sexual: Papeles, escenas y secuencias. Buenos Aires: Clacso.

Jones, D. (2010b). Diálogo entre padres y adolescentes sobre sexualidad: Discursos morales y médicos en la reproducción de la desigualdad de género.Interface-Comunic., Saude, Educ., 14 (32), pp. 171-182. Doi: 10.1590/S1414-32832010000100014.

Jones, D. (2010c). Sexualidades adolescentes, amor, placer y control en la Argentina contemporánea. Buenos Aires: Clacso.

Kajula, L., Sheon, N., De Vries, H., Kaaya, S. \& Aarø, L. (2014). Dynamics of ParentAdolescent Communication on Sexual Health and HIV/AIDS in Tanzania. AIDS \& Behavior, 18 (1), pp. 69-74. Doi: 10.1007/ s10461-013-0634-6.

Lavielle-Sotomayor, P., Jiménez-Valdez, F., Vázquez-Rodríguez, A., AguirreGarcía, M. C., Castillo-Trejo, M. \& VegaMendoza, S. (2014). Impacto de la familia en las conductas sexuales de riesgo de los adolescentes. Revista Médica del Instituto Mexicano del Seguro Social, 52 (1), pp. 3843.

López, E., Murgui, S., Musitu, G. \& Moreno, D. (2008). Clima familiar, clima escolar y satisfacción con la vida en adolescentes. Revista Mexicana de Psicología, 25 (1), pp. 119-128.

Lozano, M., Consuelo, P. \& Solís, D. (2009). El entorno familiar y escolar en la construcción de significado y sexualidad en los jóvenes de Guadalajara. La Ventana, 29, pp. 146-183.

Manu, A. A., Mba, C. J., Asare, G. Q., OdoiAgyarko, K. \& Asante, R. K. (2015). Parent-child communication about sexual and reproductive health: Evidence from the BrongAhafo region, Ghana. Reproductive Health 12(16), pp. 27372746. Doi:10.1186/s12978-015-0003-1. 
Molina, D., Torrivilla, I. \& Sánchez, Y. (2011). Significado de la educación sexual en contexto de diversidad de Venezuela. Electronic journal of research in education psychology, 9 (1), pp. 415-444.

Oglea, S., Glasiera, A. \& Rileya, S. (2008). Communication between parents and their Children about sexual. Health. Contraception, 77, pp. 283-288. Doi: 10.1016/j.contraception.2007.12.003.

Profamilia (2010). Encuesta Nacional de Demografia y Salud (ENDS).

Recuperado de: http://encuestaprofamilia. com/

Santiago, J., Gómez, J.,Etchebarne, I. \& Roussos, A. (2011). El método de investigación cualitativa consensual: Una herramienta para la investigación en psicología clínica. Anuario de Investigaciones. Facultad de Psicología UBA, 18, pp. 47-56.

Seloilwe, E. S., Magowe, M. M., Dithole, K. \& Lawrence, J. S. (2015). Parent and youth communication patterns on HIV and AIDS, STIs and sexual matters: Opportunities and challenges. Journal of Child and Adolescent Behavior, 3(3), pp. 203-213. Doi:10.4172/2375-4494.1000203

Sevilla, T. M. \& Orcasita, L. T. (2014). Abordaje de los procesos de comunicación familiar. Aspectos clave en la construcción de la sexualidad en adolescentes. En A. F. Uribe (ed.) Salud, comunicación y familia, (pp. 113-148). Bucaramanga: Universidad Pontificia Bolivariana.

Sevilla, T., Sanabria, J. P., Orcasita, L. T. \& Palma-García, D. M. (2016). Consistencies and Discrepancies in Communication about Sexuality between Parents and Children Teenagers. Paideia (RibeirãoPreto), 26(64), pp. 139-147.

Shiferaw, K., Getahun, F. \& Asres, G. (2014). Assessment of adolescents' communication on sexual and reproductive health matters with parents and associated factors among secondaryand preparatory schools' students in Debremarkos town, North West Ethiopia. Reproductive Health, 11(1), pp. 2-7. Doi: 10.1186/1742-4755-11-2.

Swain, C, R., Ackerman, L. K. \& Ackerman, M. A. (2006). The influence of individual characteristics and contraceptible beliefs on parents-teen sexual communications: A structural mode. Adolesc Health, 38(6), pp. 753-759.

Uribe, A. F. \& Rodríguez, L. (2014). Sexualidad y comunicación entre padres e hijos. Una revisión teórica. En A. F. Uribe (ed.) Salud, comunicación y familia, (pp. 83-107). Bucaramanga: Universidad Pontificia Bolivariana.

Vargas, E., Henao, J. \& González, C. (2007). Toma de decisiones románticas $\mathrm{y}$ reproductivas en la adolescencia. Revista Acta Colombiana de Psicología, 10, pp. 49-63. 